\title{
Norois
}

Environnement, aménagement, société

197 | 2005/4

Varia

\section{Dépoldériser dans le bassin d'Arcachon? Réactions sociales et propositions de gestion}

To give land back to the sea in the Arcachon Basin? Public reactions and management propositions

\section{Claire Marcadet et Lydie Goeldner-Gianella}

\section{OpenEdition}

\section{Journals}

Édition électronique

URL : https://journals.openedition.org/norois/288

DOI : $10.4000 /$ norois. 288

ISBN : 978-2-7535-1545-1

ISSN : $1760-8546$

Éditeur

Presses universitaires de Rennes

\section{Édition imprimée}

Date de publication : 1 décembre 2005

Pagination : 23-35

ISBN : 978-2-7535-0238-3

ISSN : 0029-182X

\section{Référence électronique}

Claire Marcadet et Lydie Goeldner-Gianella, « Dépoldériser dans le bassin d'Arcachon ? Réactions sociales et propositions de gestion », Norois [En ligne], 197 | 2005/4, mis en ligne le 15 décembre 2008, consulté le 13 janvier 2022. URL : http://journals.openedition.org/norois/288 ; DOI : https://doi.org/ $10.4000 /$ norois. 288

Ce document a été généré automatiquement le 13 janvier 2022.

(c) Tous droits réservés 


\title{
Dépoldériser dans le bassin d'Arcachon? Réactions sociales et propositions de gestion
}

\author{
To give land back to the sea in the Arcachon Basin? Public reactions and \\ management propositions
}

Claire Marcadet et Lydie Goeldner-Gianella

\section{NOTE DE L'ÉDITEUR}

Cet article a été reçu le 15 février 2005 et définitivement accepté le $1^{\mathrm{er}}$ juin 2005.

Les auteurs remercient le Conservatoire du Littoral et tout particulièrement Isabelle Kisielewski (délégation Aquitaine) pour son soutien, ses informations et son appui à l'obtention d'un stage.

1 En Europe occidentale, les premières dépoldérisations remontent aux années 1980. Leurs impacts physiques restent insuffisamment étudiés, mais c'est encore plus vrai des réactions sociales qu'engendre ce retour de la mer, alors qu'elles expliquent largement les échecs ou les succès de cette politique. Nous avons ainsi étudié la perception de la dépoldérisation dans le bassin d'Arcachon, où une brèche s'est produite dans la digue de mer de l'ancien polder piscicole de Graveyron, en décembre 1996. Cette brèche accidentelle devrait vraisemblablement être maintenue à l'avenir, par le Conservatoire $\mathrm{du}$ Littoral, pour des raisons tenant autant au contexte eustatique (De La Fons et Dejouhanet, 2004) qu'aux coûts engendrés par une complète reconstruction. Mais une telle décision pourrait soulever l'hostilité d'une partie des riverains et des promeneurs de Graveyron.

2 C'est en effet ce qu'a montré une enquête d'opinion conduite au printemps 2004 auprès de 200 personnes. Cette enquête avait trois objectifs : connaître la position du public sur l'aménagement du polder et cette dépoldérisation, tenter de comprendre les réactions ainsi enregistrées et établir le profil socio-géographique des personnes les 
plus défavorables au processus de dépoldérisation. Les réponses obtenues ont été dépouillées, analysées et comparées à des résultats d'autres travaux portant sur la question. Elles laissent surtout à penser que l'information de la société civile sera ici capitale pour que le Conservatoire du Littoral puisse faire comprendre et accepter le retour partiel de la mer, dans un site autrefois exploité et à la forte valeur patrimoniale.

\section{Dépoldériser en Europe occidentale et dans le bassin d'Arcachon}

\section{La dépoldérisation en Europe occidentale}

3 Après dix siècles de conquête sur la mer, l'Europe occidentale s'est lancée dans la dépoldérisation à partir des années 1980. Mais ce retour délibéré de terres à la mer a surtout pris une grande ampleur en Grande-Bretagne : on compte actuellement autant de dépoldérisations achevées ou en cours sur les seules côtes britanniques que sur l'ensemble des autres côtes d'Europe occidentale, soit une vingtaine environ dans chaque cas (Goeldner-Gianella et Imbert, 2005). Mais au total, ces entreprises ne couvrent encore que 30 à $40 \mathrm{~km}^{2}$, soit moins de $1 \%$ des $15000 \mathrm{~km}^{2}$ de polders maritimes et estuariens que compte l'Europe. Il existe des degrés gradués de remise en eau des polders, n'entraînant pas systématiquement leur élimination. On peut ainsi permettre un retour limité de la mer en installant dans la digue de mer des écluses, des portes à marée ou des canalisations. Mais on peut également créer une ou plusieurs brèches dans cette digue, en contrôlant plus ou moins les processus hydro-géomorphologiques associés. Enfin, on peut opérer un véritable démantèlement de la digue de mer, comme en Angleterre ou en Allemagne.

En règle générale, les dépoldérisations concernent des terres agricoles en déprise et ont pour objectif de recréer l'écosystème détruit par l'endiguement antérieur, qui s'était traduit par un assèchement et une désalinisation du milieu. La finalité est double: augmenter la biodiversité floristique et faunistique et créer de nouveaux lieux d'accueil pour l'avifaune, notamment migratrice. Si toutes les dépoldérisations pratiquées en Europe occidentale s'attachent à cet objectif de restauration, celles expérimentées en Grande-Bretagne s'avèrent plus complexes, car elles relèvent de la politique défensive du managed realignment. Celle-ci vise à augmenter l'efficacité et la pérennité des digues maritimes en recréant des habitats, tels que les prés salés, capables de jouer un rôle protecteur durant les tempêtes - cette recréation d'habitats pouvant justement s'opérer par un démantèlement de digue (Anonyme-b, 2002).

\section{L'importance du contexte social de la dépoldérisation}

Pour qu'une dépoldérisation réussisse, il convient de tenir compte de l'ensemble des paramètres physiques extérieurs et intérieurs aux sites qu'on souhaite rendre à la mer (French, 2001), mais il faut également s'interroger sur le contexte socio-économique favorisant ou non ce processus. Une première analyse de ce contexte social, réalisée à partir de vingt cas pris dans cinq pays d'Europe occidentale, a montré que certains facteurs avaient facilité ces entreprises (Goeldner-Gianella, 2004). Il s'agit, dans $80 \%$ des cas, de financements publics permettant l'opération. Dans $60 \%$ des cas 
apparaissent des facteurs variés, d'ordre foncier, juridique et scientifique, comme le rachat des terres par l'organisme opérant la dépoldérisation, l'existence d'une législation environnementale favorable à la restauration des marais, la forte implication de la communauté scientifique sous forme de conseil, d'expérimentations ou l'engagement d'une réflexion sur l'élévation du niveau de la mer. Par ailleurs, dans un tiers des cas, le fait que la population locale ait été informée et ait participé au processus de décision et à la gestion du site dépoldérisé a très nettement facilité la mise en œuvre de ces projets. Ce fut le cas en Angleterre (Norfolk, Wash et Humber), mais aussi à Noirmoutier ou en Frise néerlandaise. Une enquête récemment conduite en Grande-Bretagne auprès des acteurs concernés par la politique du managed realignment a donné des résultats tout à fait similaires sur l'importance de l'implication de la population (Anonyme-b, 2002). La participation de la société civile semble bien être l'une des conditions de l'acceptation et de la réussite des dépoldérisations.

Mais pour faire participer le mieux possible la société civile à ce processus, il faudrait en premier lieu mieux comprendre les représentations qu'elle s'en forge. Or, les enquêtes d'opinion menées jusqu'à présent sur ce thème sont encore peu nombreuses. On peut évoquer celles de L. B. Myatt et al., conduites en Angleterre dans les sites de Brandcaster, d'Orplands et de Freyston Shore (Myatt et al., 2003a, 2003b, 2003c), de même que celles récemment menées en France : en Bretagne (Goeldner-Gianella et Imbert, 2005), à Noirmoutier (Barbe, 2005) et dans le bassin d'Arcachon. Cette dernière enquête fait suite à une expertise demandée par le Conservatoire du Littoral sur le futur risque de submersion de l'un de ses polders dans le bassin d'Arcachon (GoeldnerGianella et al., 2003). La méthode et les résultats de cette enquête sont détaillés cidessous et reprennent des travaux de recherche effectués dans le cadre d'une maitrise de géographie (Marcadet, 2004).

\section{Le domaine de Graveyron : état des lieux et des enjeux}

7 Cette enquête d'opinion a porté sur le domaine de Graveyron, polder de 135 ha situé sur la rive orientale du bassin d'Arcachon, au nord du delta de la Leyre, dans la commune d'Audenge (fig. 1). C'est la combinaison de plusieurs activités, depuis le XVIII siècle, qui a conféré toute son originalité à ce site : pisciculture dans des réservoirs, céréaliculture et pâture dans les prairies. Les réservoirs à poissons, de profondeurs variables, étaient alimentés en eau de mer et en alevins par des écluses (Labourg, 1985). Mais à partir de 1960, le déclin de l'aquaculture s'est amorcé dans le bassin d'Arcachon. À Graveyron, le milieu s'est embroussaillé, le paysage s'est fermé et les digues se sont déteriorées, tandis que le site était colonisé par de nouvelles espèces. Il offre aujourd'hui une grande diversité d'habitats (Anonyme-a, 2002): prairies humides, phragmitaies, jonçaies et anciens réservoirs à poissons, qui constituent des milieux favorables à l'alimentation de la faune (vison d'Europe, loutre) et surtout des oiseaux (milan noir, busard des roseaux). Cette richesse est reconnue (ZNIEFF 1) et bénéficie de plusieurs protections (zone ND du POS, Site Inscrit,site Natura 2000 et propriété du Conservatoire du Littoral depuis décembre 1998). Mais la formation d'une brèche dans la digue frontale (photos 1 et 2), lors d'une tempête en décembre 1996, a aussi permis à un schorre de se reconstituer sur une dizaine d'hectares, dans la partie ouest de Graveyron appelée «La Pointe» (photo 3 et fig. 1). Cette réouverture accidentelle a incité le Conservatoire du Littoral et le Conseil Général de Gironde, gestionnaire du site, à 
engager une réflexion sur le devenir de cette Pointe et de l'ensemble du domaine de Graveyron (Kisielewski, 2002) : faut-il combler la brèche ou la maintenir, ou bien aller jusqu'à dépoldériser la totalité du domaine? Quels seraient les enjeux d'une telle entreprise?

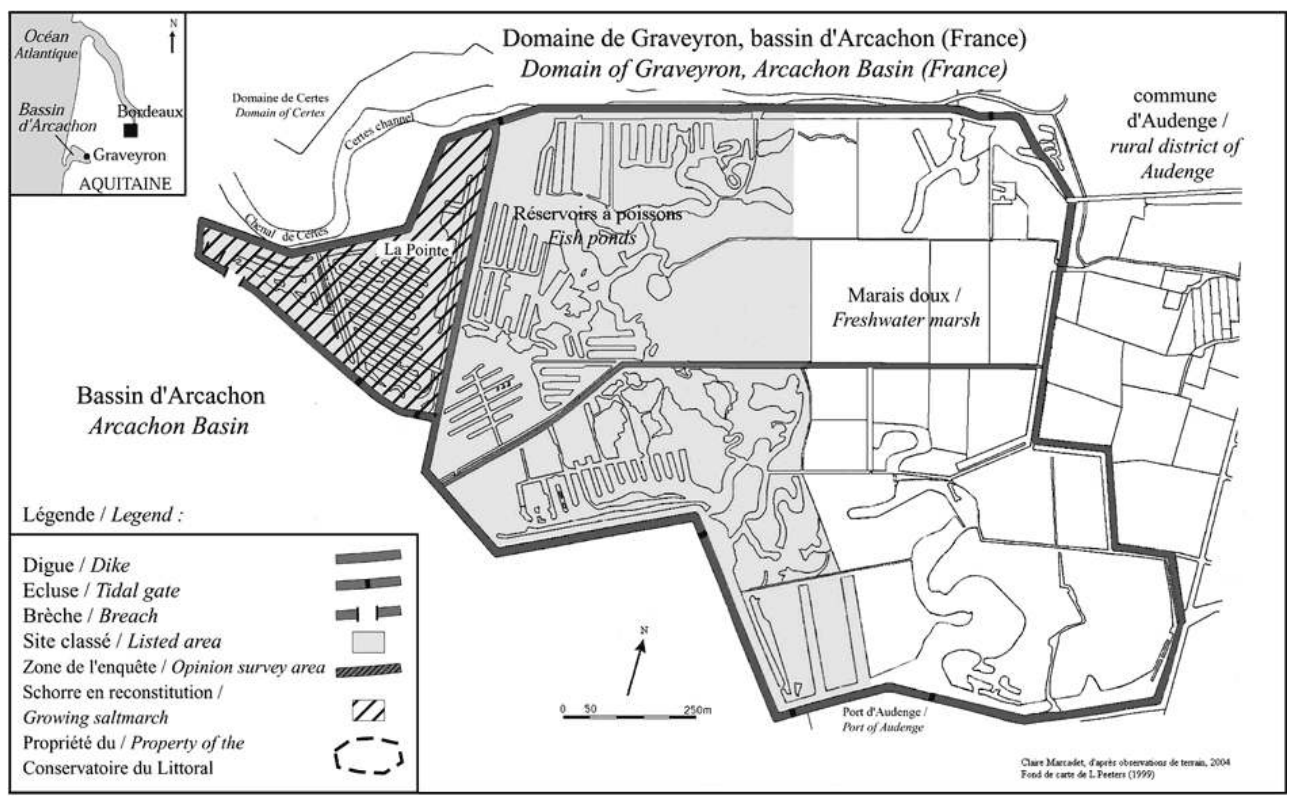

Figure 1 : Localisation et présentation du site d'étude Localization and presentation of the study area

D'un point de vue écologique, cette dépoldérisation partielle accentue la richesse du bassin d'Arcachon : elle permet la progression des prés salés - qui ont reculé dans le bassin entre 1964 et 1988 (Soriano-Sierra, 1992), alors qu'ils comptent parmi les plus riches de France sur le plan floristique (Géhu et Géhu-Franck, 1982) - et est favorable à l'avifaune, très nombreuse dans le bassin qui représente l'une des cinq zones humides les plus fréquentées de France. Enfin, le site dépoldérisé abrite une espèce rare et protégée à l'échelle régionale (le Triglochin barrelieri). L'alternance des marées dans la Pointe a aussi permis l'élimination de Baccharis halimifolia - plante invasive qui banalise le milieu et ferme le paysage - favorisant ainsi le travail du conseil général de Gironde qui essaie de réduire sa présence par un girobroyage des racines dans le reste du domaine. La présence d'un schorre est par ailleurs appréciée par l'Entente Interdépartementale pour la Démoustication: comme l'eau n'y stagne pas, les moustiques n'y déposent pas leurs œufs et l'EID n'a pas à traiter cette partie du site. Enfin, l'enjeu est aussi largement social car le domaine de Graveyron, ouvert au public, est fréquenté par la population locale et les touristes. Cependant, on ignore leur opinion sur cette réouverture. On peut toutefois supposer que les habitants d'Audenge et des environs y seraient hostiles, puisqu'ils considèrent que le domaine de Graveyron fait partie du patrimoine local et qu'ils l'utilisent comme lieu de promenade. 


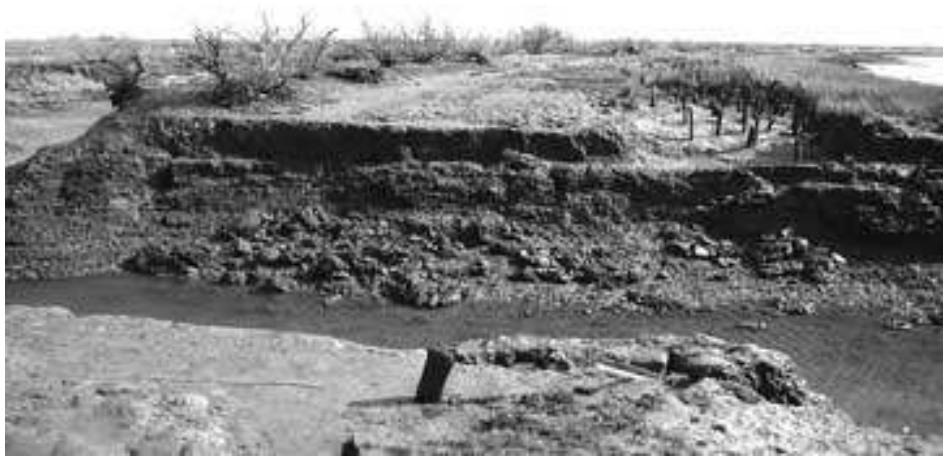

Photo 1 : Brèche de Graveyron à marée descendante, mars 2004 (cl. C. Marcadet) The breach of Graveyron during falling tide, march 2004

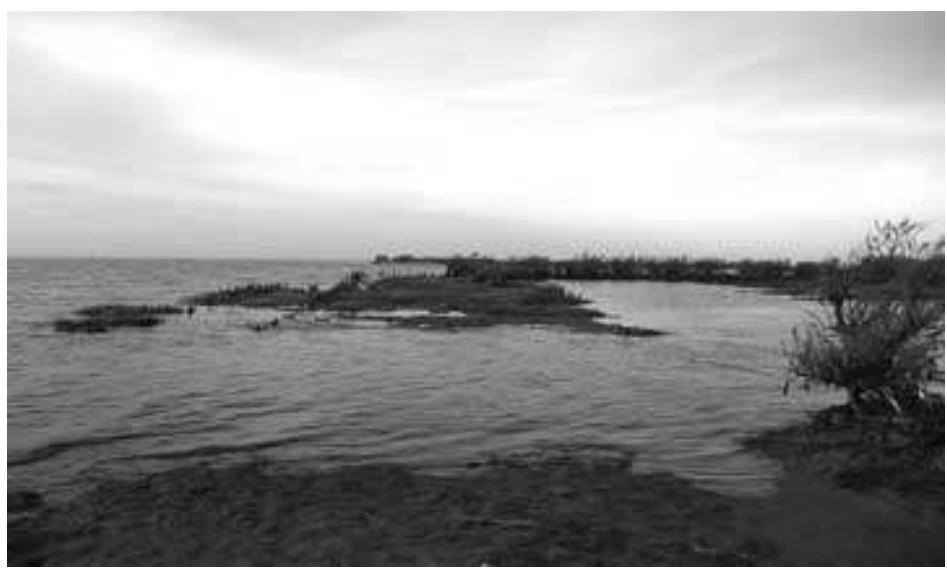

Photo 2 : Brèche de Graveyron à marée haute, décembre 2003 (cl. C. Marcadet) The breach of Graveyron at high tide, december 2003



Photo 3 : Le secteur rendu à la mer et son schorre, sept ans après la formation d'une brèche, mars 2004 (cl. C. Marcadet)

The area restored to the sea and its saltmarsh, seven years after a breach had occurred, march 2004 


\section{L'enquête de Graveyron : méthodes et résultats}

\section{Protocole d'enquête}

9 En se basant sur des travaux scientifiques antérieurs, plusieurs hypothèses de départ ont été émises pour aider à la conception du questionnaire d'enquête. La première de ces hypothèses est que la population n'a pas d'avis réel sur le thème de la dépoldérisation, du fait d'un manque de connaissances sur les marais maritimes et sur les impacts d'un retour à la mer. Cette explication a été avancée dans le cas du polder de Sables-d'Or-les-Pins, en Bretagne (Goeldner-Gianella et Imbert, 2005). Notre seconde hypothèse est que le polder est utilisé comme lieu de détente «naturel » où l'on pratique des loisirs à pied, ce qui laisse à penser qu'un retour du site à la mer serait refusé par les usagers. Notre enquête a consisté en un questionnaire oral standardisé comprenant des questions fermées et quelques questions ouvertes. Nous avions choisi d'interroger 200 personnes majeures, la moitié à Graveyron même et l'autre moitié aux domiciles les plus proches, afin d'obtenir un échantillon suffisamment large et diversifié. Mais étant donné le nombre élevé de résidences vides, lors de notre passage à Audenge, seuls 33 questionnaires ont été remplis chez des particuliers ${ }^{1}$. Les 167 autres répondants ont été principalement interrogés sur la digue sud du polder, entre le port et la plage d'Audenge (fig. 1). L'enquête s'est déroulée en 3 semaines, en mars et avril 2004, aussi bien le week-end que durant la semaine. Sur les 200 répondants, on dénombre quasiment autant d'habitants $\mathrm{du}$ bassin que de touristes (personne domiciliée hors des communes riveraines du bassin) et autant de femmes que d'hommes. Les tranches d'âge sont plus inégales puisque les 40-59 anssontlégèrementmajoritaires ( $40 \%$ ) par rapport aux jeunes adultes (18-39 ans) et aux personnes de plus de 60 ans.

\section{Fréquentation et usages du polder}

10 À la première question du questionnaire « Connaissez-vous le site de Graveyron ? », on obtient à peu près autant de réponses positives que de réponses négatives. Cela signifie que $50 \%$ des répondants ne savent pas qu'ils se trouvent dans le polder de Graveyron, alors qu'ils ont été interrogés sur sa digue! Sur ces 95 personnes, 61 sont des touristes (résidant hors du bassin d'Arcachon), 22 sont des habitants d'une commune du bassin (hors Audenge) et 12 sont des habitants d'Audenge (dont 6 ont été interrogés à leur domicile). Les touristes viennent en grande majorité pour la première fois et cela montre qu'ils n'ont pas lu le panneau à l'entrée du domaine indiquant le nom du site. Par contre, pour les habitants du bassin et d'Audenge, qui viennent plus régulièrement (certains depuis plus de 15 ans), cela est plus étonnant car ils sont supposés avoir une meilleure connaissance de leur environnement local. La plupart d'entre eux pensait d'ailleurs se trouver sur le domaine voisin de Certes (fig. 1)... Pourtant, bien que le nom de Graveyron soit mal connu, le site, lui, est connu en tant que tel et est relativement fréquenté. Les deux tiers des répondants ne viennent cependant que quelques fois par an (un tiers d'entre eux venant d'ailleurs pour la première fois). Mais ceux qui fréquentent le polder plus régulièrement viennent 1 à 2 fois par mois (19\%), voire 1 à 2 fois par semaine (15\%). Sur ces personnes fréquentant très souvent Graveyron les deux tiers résident à Audenge, $22 \%$ dans une commune du bassin (Biganos, Marcheprime et Lège) et $10 \%$ dans l'agglomération bordelaise. 
L'enquête confirme notre hypothèse selon laquelle le polder est utilisé comme lieu de détente «naturel » où l'on pratique des loisirs à pied, puisque les neuf dixièmes de la population interrogée pratiquent au moins la promenade à pied, un quart la découverte et l'observation des animaux et des plantes et un cinquième un sport (footing, vélo) ${ }^{2}$. La pêche à pied et la chasse à la tonne restent des activités minoritaires ne concernant que $5 \%$ et $2,5 \%$ des usagers.

12 Lorsqu'on interroge ces personnes sur le devenir possible de Graveyron, celles-ci formulent des souhaits simples (fig. 2) : mieux "découvrir » le site (32\%) et surtout conserver son caractère naturel ou en faire une réserve naturelle (49\%). Ce double intérêt pour la nature pourrait donc laisser supposer que la population ne serait pas complètement hostile à une dépoldérisation du site, répondant à des objectifs écologiques et paysagers. Mais l'on peut aussi comprendre ces propositions comme un souhait de conserver le site dans son état actuel - c'est-à-dire celui d'un marais certes riche sur le plan naturel, mais demeurant endigué3 ${ }^{3} .$.

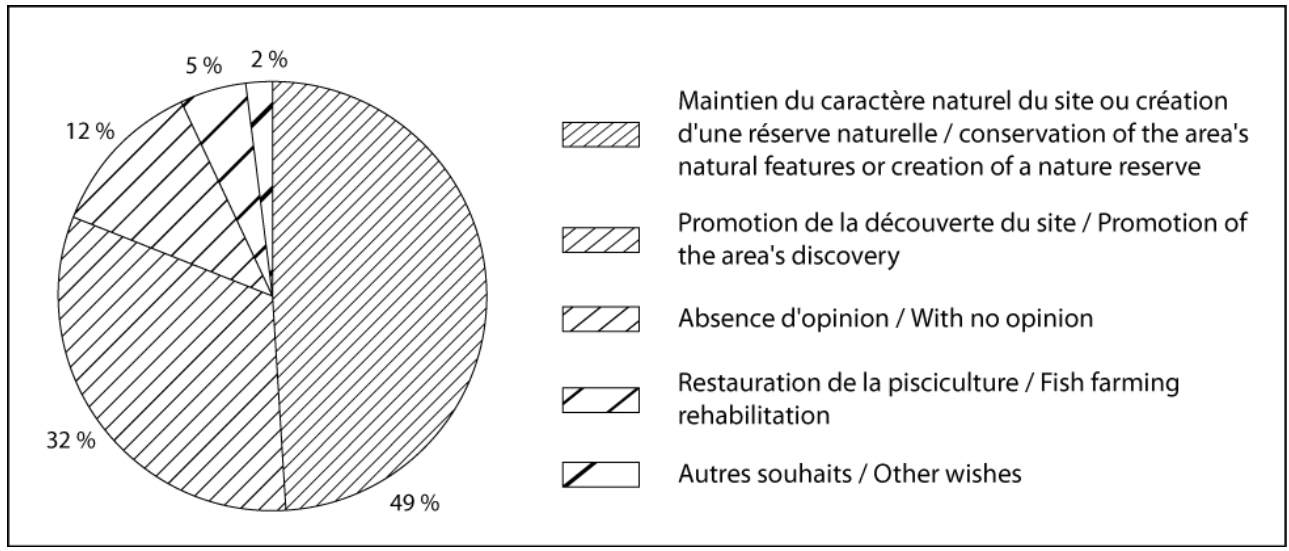

Figure 2 : «Étant donné que la piscitulture a été abandonnée, quelle nouvelle vocation le polder de Graveyron pourrait-il avoir?»

"As fish farming has been abandoned, which sort of calling could be developed in the polder of Graveyron?"

\section{Le refus d'une dépoldérisation}

Concernant plus précisément la brèche apparue dans la digue de la Pointe en 1996, seuls 43 répondants disent avoir observé des changements dans le site au fil du temps et, parmi ceux-là, seuls 7 mentionnent explicitement cette brèche. Il faut sans doute relier cette faible proportion au fait qu'un quart des répondants vient pour la première fois à Graveyron et qu'en tout ils sont près de deux tiers à connaître le site depuis moins de 8 ans (date d'apparition de la brèche). Au premier abord la brèche n'apparaît donc pas comme une préoccupation importante et il est possible que la majorité des répondants ne se soit pas promenée jusque-là, mais cela ne l'empêche pas d'avoir une opinion sur la question d'une dépoldérisation.

En effet, la majorité de la population interrogée est opposée à la réouverture du polder (fig. 3) : à la question "Quelle action pourrait être envisagée pour le secteur de la Pointe? ", les deux tiers des personnes proposent soit de " combler la brèche ", soit de " combler la brèche, reconstruire et rehausser la digue intermédiaire ». Même si la part des personnes sans opinion est assez importante (un quart des répondants), notre hypothèse de départ selon laquelle la population n'avait pas d'avis sur cette 
réouverture n'est donc pas validée. La réouverture de la Pointe est même loin de laisser indifférent.

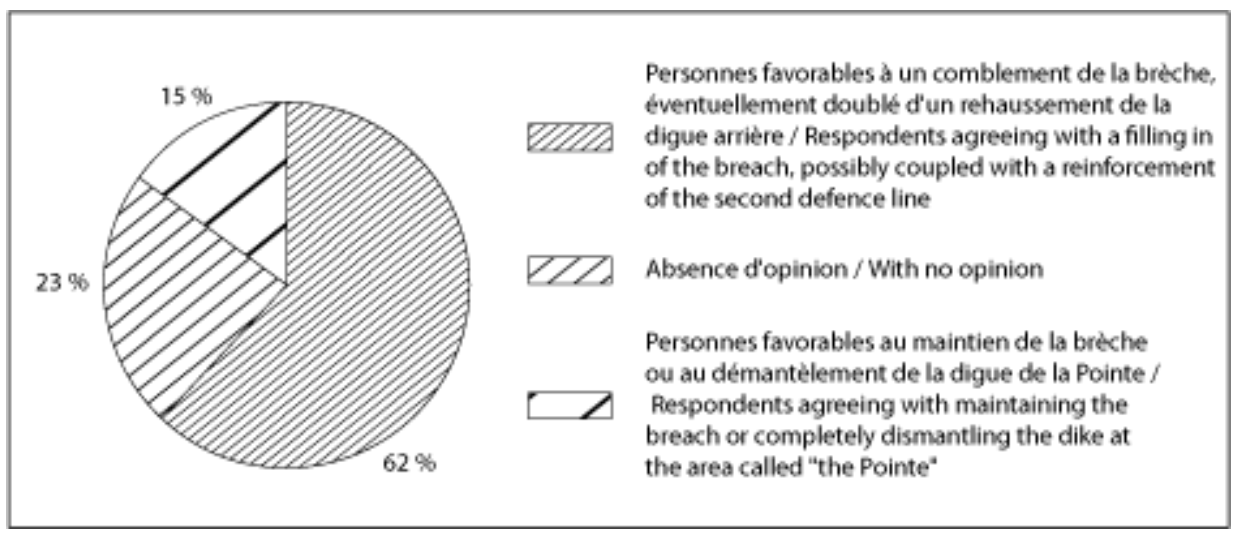

Figure 3 : «Quelle action pourrait être entreprise pour le secteur de la Pointe?»

"Which measures could be taken for the area called 'the Pointe'?"

15 Cette opposition devient plus forte encore lorsque l'enquêteur évoque la possibilité d'une dépoldérisation complète du site (et pas seulement de la Pointe) et envisage ses conséquences sur divers plans. Dans ce cas, 80 à $90 \%$ des personnes interrogées refuseraient un tel abandon à la mer pour des raisons financières et écologiques, mais plus encore paysagères et culturelles. L'opposition à une telle mesure de restauration de la nature n'offre donc aucune ambiguïté et devrait être prise en compte par le Conservatoire du Littoral. On peut toutefois distinguer deux niveaux d'acceptation dans la dépoldérisation de Graveyron: la réouverture accidentelle d'une petite partie du domaine semblerait mieux acceptée qu'une dépoldérisation totale et organisée, qui serait mal perçue par la quasi-totalité de la population.

\section{Éléments d'explication et propositions de gestion}

L'enquête menée à Graveyron et des comparaisons avec d'autres enquêtes portant sur ces questions ont permis de dégager quelques éléments d'explication.

\section{Une faible connaissance des marais maritimes et des polders}

17 En premier lieu, l'opposition à la réouverture de la Pointe peut s'interpréter comme la peur de voir disparaitre ou changer Graveyron. En effet, pour justifier leur position, les personnes favorables au comblement de la brèche - c'est-à-dire opposées à une dépoldérisation - proposent plutôt (dans des questions ouvertes) de "protéger ou conserver le polder", d'" éviter sa destruction», de "restaurer le sentier, la promenade $"$, de conserver le polder car "il a toujours été là ». Mais ces réponses reflètent aussi une mauvaise connaissance du milieu. Ainsi, $12 \%$ de ces personnes pensent que l'eau salée est néfaste pour le polder, alors qu'elle a toujours été un élément important du domaine, contribuant au renouvellement des eaux et à la régulation de la salinité pour la pisciculture. Les écluses à marée en sont d'ailleurs le témoignage visible.

Ce manque de connaissances sur le milieu et sur les effets d'une dépoldérisation est confirmé par le fait qu'un tiers des personnes interrogées n'a, de toute façon, pas d'avis sur les conséquences de la brèche et que les autres réponses recèlent une connotation 
largement négative (fig. 4). Plus de la moitié des personnes interrogées estime ainsi que la réouverture pourrait conduire à l'érosion des digues et à la disparition du polder. La modification de l'écosystème est énoncée dans ces réponses de façon assez neutre (difficultés des répondants pour déterminer si cela est positif ou non), et l'intrusion de l'eau salée est de nouveau vécue comme une mauvaise chose. D'une façon générale, que la réponse soit connotée de façon négative ou positive, elle ne mentionne souvent qu'une seule des multiples conséquences de la réouverture. La population ne disposant pas des informations nécessaires pour appréhender le phénomène sous tous ses angles, il est alors perçu comme une menace. En abordant plus précisément les impacts de la réouverture sur le milieu naturel («Selon vous, ce retour à la mer est-il favorable au milieu naturel?»), aucune opinion ne ressort franchement puisque l'on obtient un tiers de chaque réponse possible («oui », «non », et «sans opinion »). Cette situation semble suggérer un manque général de connaissances sur le fonctionnement écologique des marais maritimes.

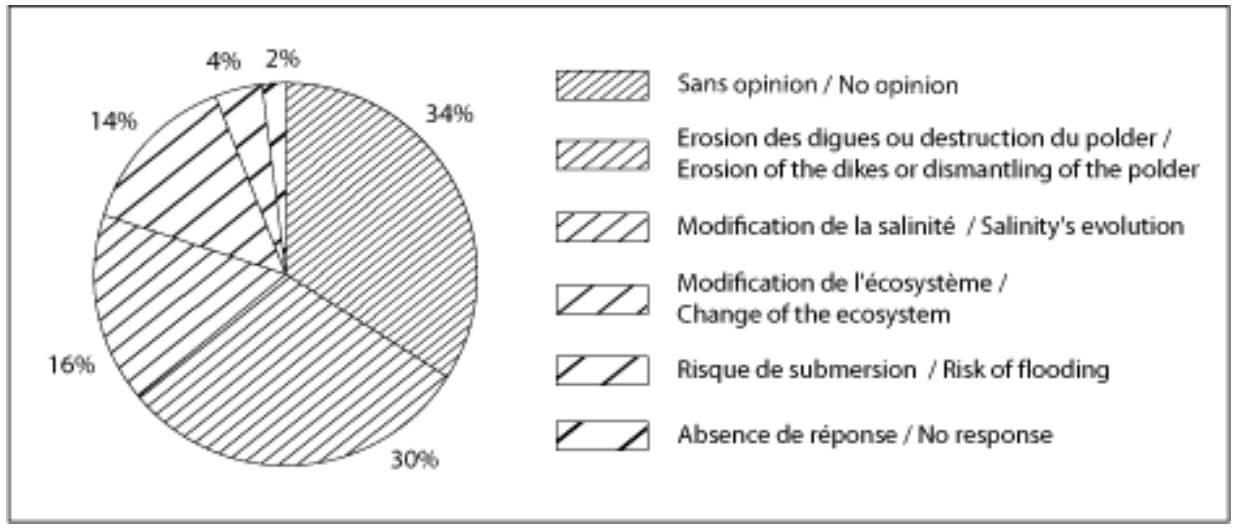

Figure 4 : «En 1996, une brèche s'est formée dans la digue de la Pointe. Depuis, l'eau de mer rentre dans le polder au gré des marées. D'après vous, qu'elles en sont les conséquences ? » "In 1996 an accidental breach occured in the sea wall of the Pointe, allowing the sea waters to enter the polder. If you had an opinion, about which consequences would you think?"

De la même façon, les "polders » sont assez mal connus, puisqu'à la question «Connaissez-vous le mot polder?» on obtient $40 \%$ de «non». Parmi ceux qui connaissent le terme, la majorité parle de la «Hollande », d'» une terre gagnée sur la mer » et de "digues». Mais une part importante ne parvient pas à définir le terme et beaucoup de réponses n'ont même aucun sens : plusieurs d'entre elles font vaguement référence à l'eau (« retenue d'eau ", «terre avec de l'eau », « eau qui rentre dans les terres») ou sont complètement inadaptées («trou dans l'eau», «endroit hors industries ", « nature », « retenue d'eau » ou encore " terre-île »). Au final, seul un tiers de ceux qui affirment connaître la notion de polder s'en fait une idée à peu près claire et serait ainsi susceptible de comprendre les effets d'une poldérisation ou d'une dépoldérisation sur le milieu naturel. Cette méconnaissance est confirmée par le fait que $40 \%$ des sondés pensent que Graveyron est un site "naturel», alors que l'enquêteur leur a signalé, lorsqu'ils n'étaient pas capables de définir le terme de "polder", qu'il s'agissait d'un aménagement réalisé par l'Homme. Cette mauvaise connaissance des milieux de polders et de marais, également observée dans d'autres enquêtes (Goeldner-Gianella et Imbert,2005), pourrait être l'une des raisons expliquant l'hostilité de la société civile à la dépoldérisation. 


\section{Profil des personnes défavorables à une dépoldérisation} divers éléments en relation et vérifié par un test statistique (chi2) la validité des résultats obtenus. Nous avons ainsi établi des liens significatifs entre l'opinion sur la réouverture de la Pointe et l'âge des personnes interrogées, leur lieu de résidence et l'ancienneté de leur fréquentation du site.

21 Les plus de 60 ans sont de toute évidence les plus favorables au comblement de la brèche, contrairement aux plus jeunes (fig. 5). Il y a donc un effet d'âge et de génération, qui correspond bien aux observations faites par L. B. Myatt et al. (2003a), lors de l'enquête sur la dépoldérisation de Brancaster (Grande-Bretagne). L'opinion sur la réouverture du polder a aussi un lien avec l'ancienneté de la fréquentation : plus on fréquente Graveyron depuis longtemps, plus on est favorable au comblement de la brèche. En effet, ceux qui connaissent le polder depuis moins de 13 ans (incluant ceux venant à Graveyron pour la première fois) sont moins opposés à la réouverture que ceux qui le fréquentent depuis plus de 13 ans $^{5}$. Ce résultat est là encore similaire à celui que d'autres chercheurs ont observé, notamment à Sables-d'Or-les-Pins (GoeldnerGianella et Imbert, 2005).

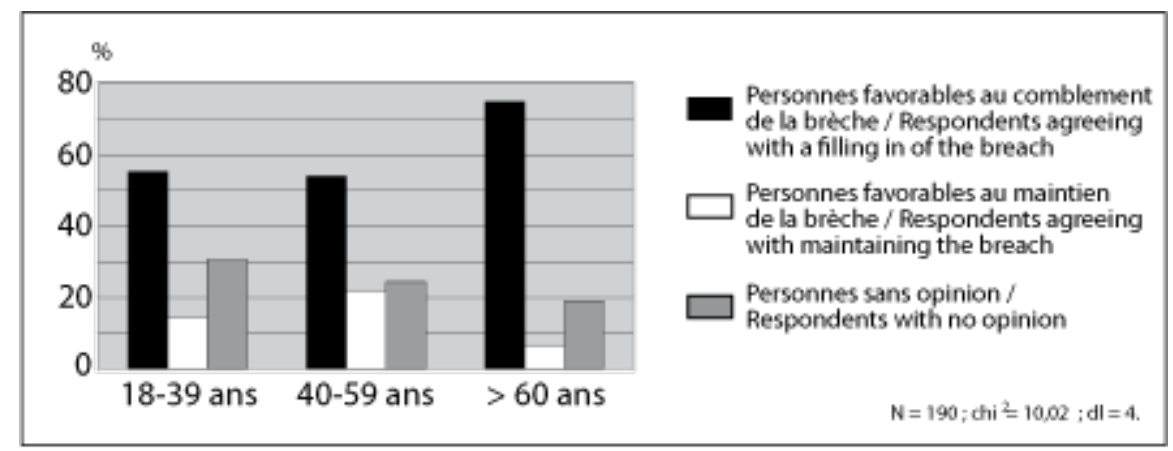

Figure 5 : Opinions sur la dépoldérisation de Graveyron et âge des personnes interrogées Frequency of opinions on the de-polderisation at Graveyron by age of respondents

Enfin, on peut aussi souligner les liens qui existent entre l'opposition à la dépoldérisation et le lieu de résidence : plus le domicile est proche de Graveyron, plus on est favorable au comblement de la brèche et inversement, plus on en habite loin, moins on a d'avis à ce sujet (fig. 6). Ainsi, les habitants d'Audenge seraient plus soucieux de la conservation de la promenade et du polder, qui constitue pour eux un lieu de nature et loisirs, que ceux qui n'y vivent pas. Graveyron fait partie du patrimoine naturel et historique local, et les habitants des communes du bassin d'Arcachon mais surtout d'Audenge y sont attachés et veulent le conserver. Il faut souligner le fait que la population n'est pas inquiète par rapport à la possibilité de submersion des habitations par la mer, puisque seuls $13 \%$ pensent que «le maintien d'une ouverture ou l'enlèvement complet de la digue de la Pointe pourraient constituer un danger pour la population d'Audenge ». L'opposition à la réouverture de la part des riverains ne se fait donc pas dans un souci de se prémunir contre les submersions mais bien dans l'optique de conserver le site pour ses qualités paysagères et récréatives. 


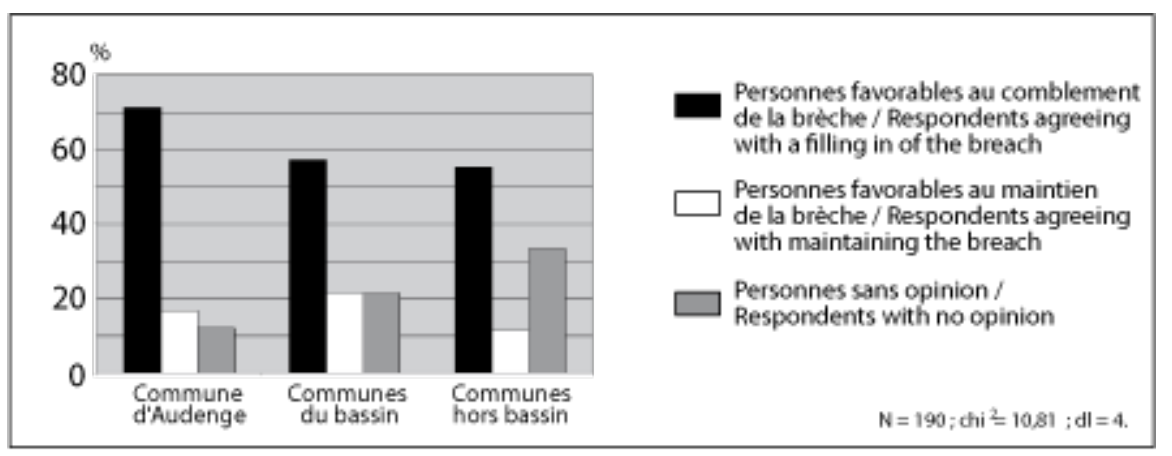

Figure 6 : Opinions sur la dépoldérisation de Graveyron et lieu de résidence des personnes interrogées Frequency of opinions on the de-polderisation at Graveyron by distance of respondents' residence

\section{Quelles propositions de gestion tirer de cette enquête?}

Après cette enquête, deux solutions apparaissent : combler la brèche pour se conformer à l'opinion du public, ou bien la maintenir tout en développant l'information sur le fonctionnement du polder et les impacts du retour à la mer, afin de favoriser la compréhension et l'acceptation de la réouverture par le public.

Si, lors de l'acquisition de Graveyron, le Conservatoire du Littoral était plutôt indécis quant à la gestion de la Pointe, l'idée de fermeture a désormais été écartée par le Conseil Scientifique du Conservatoire (Conseil Scientifique du CELRL, 2002). La reconstitution du schorre présente des intérêts écologiques, paysagers et scientifiques qu'il est difficile de remettre en question après huit ans de réouverture. Les coûts élevés qu'engendrerait la restauration de la totalité de la digue de la Pointe sont également dissuasifs (500 000 euros selon Kisielewski, 2002). Pour la partie interne de Graveyron, une dépoldérisation ne serait pas envisageable, l'objectif étant de favoriser la biodiversité d'un marais doux et les activités traditionnelles (élevage), de développer les itinéraires de promenade, et de rehausser les digues pour se prémunir contre la hausse du niveau marin. Mais si la solution de laisser la Pointe ouverte semble de plus en plus inévitable à ceux qui gèrent quotidiennement le polder, elle ne sera pas pour autant facilement acceptée. L'enquête a montré que la majorité des personnes interrogées était hostile au maintien d'une brèche qui semble néfaste pour le milieu naturel et pourrait constituer un danger pour le reste du polder. Le choix de conserver l'ouverture sur la mer devra donc être expliqué, pour éviter de donner au public l'impression que le Conservatoire du Littoral et le conseil général de Gironde laissent le site à l'abandon.

En effet, l'enquête réalisée à Brancaster (Myatt et al., 2003a) a révélé que la réticence de la population vis-à-vis de la dépoldérisation était largement due au manque d'information sur cette politique. L'information semble donc être le maître-mot de l'acceptation d'une dépoldérisation, comme l'a bien montré une autre enquête, cette fois à Noirmoutier. En 2004, $75 \%$ de la population interrogée se déclarait favorable à la submersion partielle et contrôlée du polder de Sébastopol (Barbe, 2005) ${ }^{6}$. Or, une approbation aussi massive pourrait découler du fait que les répondants avaient pu lire, avant d'être interrogés, un remarquable panneau d'information placé à l'entrée du polder. Leur parfaite connaissance de la notion de "poldérisation » et de l'avifaune présente dans le polder serait ainsi à mettre en rapport avec le contenu du panneau, très explicite à ce sujet. Si l'on compare ces réponses exactes à celles obtenues à 
Graveyron, globalement vagues et incorrectes, l'écart des connaissances se révèle étonnant. L'information fournie par ce panneau se serait donc avérée efficace : les gens auraient compris l'intérêt d'une dépoldérisation pour l'avifaune et approuveraient alors très largement l'expérience de remise en eau du polder de Sébastopol.

Un panneau de même nature, situé aux entrées du domaine de Graveyron, pourrait y expliquer la présence de la brèche (rupture lors d'une tempête, choix du Conservatoire du Littoral de la maintenir, intérêt scientifique du site) et ses conséquences (reconstitution du schorre, élimination du Baccharis halimifolia,etc.). L'enquête montre également que la population souhaiterait qu'on installe dans le polder des panneaux sur l'histoire du site, sa faune et sa flore et les itinéraires existants - à l'image de ce qui a été réalisé à l'entrée du polder de Sébastopol. Certains aimeraient participer à des visites guidées de découverte (histoire de la pisciculture, flore et faune) et recevoir des dépliants. De nouveaux aménagements, tels que des bancs en bois et des poubelles, et un meilleur entretien du sentier (éviter les trous et la boue) sont aussi demandés - bien que les sites du Conservatoire du Littoral soient en réalité des espaces naturels et non des " parcs »... Cependant, les usagers demandent à ce que ces aménagements restent légers et s'intègrent bien dans le paysage pour ne pas dénaturer le site. Une grande partie de la population vient à Graveyron pour être dans la nature et sortir de son univers urbain, mais exige le même confort qu'en ville - comme c'est souvent le cas dans d'autres sites du Conservatoire du Littoral. Il faudrait donc trouver un juste milieu entre le maintien de la réouverture sur la mer, l'information du public, les aménagements que cela nécessite et la conservation du patrimoine.

\section{Conclusion}

Pour affiner leurs choix, le propriétaire et le gestionnaire de Graveyron peuvent se référer aux résultats de cette enquête, qui montrent notamment combien l'information $\mathrm{du}$ public serait fondamentale pour faire comprendre et ensuite accepter le retour partiel de la mer dans le polder de Graveyron.

Mais il faudrait également tenir compte du devenir du site face à l'élévation du niveau de la mer. En effet, une expertise a récemment montré qu'en période de pleine mer de vive eau exceptionnelle, l'ensemble du domaine de Graveyron était submersible même sans rupture de ses digues (Goeldner-Gianella et al., 2003). Or, dans un siècle, l'élévation $\mathrm{du}$ niveau de la mer se traduira par un risque de submersion totale dès une simple pleine mer de vive eau avec légère surcote, submersion menaçant même les premières habitations d'Audenge... Pour éviter la perte du domaine, il faudra alors consolider et rehausser les digues extérieures. Mais il serait utile d'informer dès à présent la population de ce risque de submersion majeure, pour lui permettre de relativiser la perte des 10 ha qui s'est déjà produite dans la Pointe. Celle-ci comprendrait alors que, sur les plans défensifs et économiques, il est plus urgent d'intervenir dans la partie arrière du polder que dans sa partie aval, qu'on pourrait définitivement abandonner à la mer. La diffusion récente d'un atlas sur la submersion future des propriétés du Conservatoire du Littoral va d'ailleurs bien dans ce sens, montrant très explicitement qu'il y aura une submersion quasi-complète de Graveyron pouvant "affecter des installations humaines » (De La Fons et Dejouhanet, 2004).

Par ailleurs, le maintien de la brèche de Graveyron contribuerait à diversifier les paysages du polder, déjà appréciés des promeneurs, et permettrait aux scientifiques 
français de disposer d'un site d'étude de la dépoldérisation, où ils pourraient examiner ses divers impacts et mieux déterminer les paramètres physiques les plus favorables à sa réussite.

\section{BIBLIOGRAPHIE}

ANONYME-a, 2002. - Plan de gestion - Domaine de Certes et de Graveyron (33), Analyse du Patrimoine naturel et du paysage, rapport provisoire (consultable au CELRL, Bordeaux), GEREA/AVEC, $33 \mathrm{p}$. ANONYME-b, 2002. - Managed Realignment Review, R\&D Technical Report FD2008, Department for Environment, Food and Rural Affairs, Environment Agency, Flood and Coastal Defence R\&D Programme, $212 \mathrm{p}$.

BARBE (J.), 2005. - Agriculture et environnement à Noirmoutier : les destins divergents de la plaine de Barbâtre et du polder de Sébastopol, Maîtrise de Géographie, Université de Paris I, 132 p.

CONSEIL SCIENTIFIQUE DU CELRL, 2002, - Pistes de réflexion pour des orientations de gestion - domaines endigués du Delta de Leyre.

FRENCH (P. W.), 2001. - Coastal defences. Problems and solutions, edition Routledge, London, 366 p.

GeHu (J.-M.), GeHU-Franck (J.), 1982. - « Étude phytocœnotique analytique et globale de l'ensemble des vases et prés salés et saumâtres de la façade atlantique française », Bull. Ecol., vol. 13, n 4, p. 357-386.

Goeldner-Gianella (L.), Anselme (B.), Bertrand (F.), DuRAnd (P.), 2003. - Recul du trait de côte et orientations stratégiques dans le domaine de Graveyron (bassin d'Arcachon, Gironde), expertise réalisée pour le Conservatoire du Littoral dans le cadre du Programme « Impacts du changement climatique sur l'intervention du Conservatoire du Littoral », $48 \mathrm{p}$.

GOELDNER-Gianella (L.), 2004. - « Vouloir, accepter ou refuser la dépoldérisation », dans CORNETTE (J.-C.), TRIPLet (P.), Rolland (G.), Pour une approche intégrée de la protection de la nature en faveur des oiseaux d'eau / L'homme et la nature ont-ils un avenir commun dans les estuaires?, Actes de l'atelier Eurosite, SMACOPI, 22/23 avril 2004, p. 11-18.

GOELDNER-GiANELLA (L.), IMBERT (C.), 2005. - « Représentations sociales des marais et dépoldérisation. Le cas d'un marais breton », L’Espace Géographique, n 3, p. 251-265.

KISIELEWSKI (I), 2002. - Réflexion sur les domaines endigués du bassin d'Arcachon : dépoldérisation ou restauration des aménagements de protection des domaines de cartes, de Graveyron et de l'île de Malprat ?, CELRL, Délégation Aquitaine, $22 \mathrm{p}$.

LABOURG (P.-J.), 1985. - « Écologie et utilisation des zones humides du bassin d'Arcachon », Actes du Colloque Zones Humides du Littoral Aquitain, Le Teich, septembre, p. 5-28.

LA Fons (G. de), DejouHANET (L.), 2004. - Impact du changement climatique sur le patrimoine du Conservatoire du Littoral. Scénarios d'érosion et de submersion à l'horizon 2100. Atlas, Conservatoire du Littoral, $50 \mathrm{p}$. 
MARCADET (C.), 2004. - La réouverture du polder de Graveyron (bassin d'Arcachon, Gironde) : risque de submersion, réactions sociales et gestion du site, Maîtrise de Géographie, Université Paris I, 97 p.

Myatt (L. B.), SCRimshaw (M. D.), Lester (J. N.), 2003a. - « Public Perceptions and Attitudes towards a Current Managed Realignment Scheme : Brancaster West Marsh, North Norfolk, UK ", Journal of Coastal Research, vol. 19, n 2, p. 278-286.

,$- 2003 \mathrm{~b}$. - « Public perceptions and attitudes towards a forthcoming managed realignment scheme : Freiston Shore, Lincolnshire, UK », Ocean \& Coastal Management, vol. 46, p. 565-582.

-, 2003c. - « Public perceptions and attitudes towards an established managed realignment scheme : Orplands, Essex, UK », Journal of Environmental Management, vol. 68, n 2, p. 173-181.

PeETERS (L.), 1999. - Plan de gestion de Graveyron, Mémoire de DESS, UST Lille 1, 152 p.

SORIANO-SIERRA (E.-J.), 1992. - Étude écologique des marais salés du bassin d'Arcachon, structure et évolution des schorres, production et dégradation de leur végétation (microfiche), Thèse de doctorat en écologie, Université de Bordeaux I, 255 p.

\section{NOTES}

1. Sur les 33 riverains, 4 ont déclaré ne pas connaître le domaine de Graveyron (même après localisation) et 6 ne jamais s'y rendre (les questions concernant le polder ne leur ont donc pas été posées. Toutefois, ces questionnaires ont quand même été inclus dans les résultats pour montrer que tous les riverains ne se rendent pas à Graveyron). Pour cette raison, la plupart des questions auront un effectif total égal à 190 et non à 200.

2. Plusieurs activités sont parfois pratiquées (ex. : promenade à pied et sport), ce qui explique que la somme des pourcentages soit supérieure à 100 .

3. À l'exception du secteur de la Pointe.

4. Promenade interrompue par la présence d'une brèche.

5. La valeur de 13 ans est la moyenne du nombre d'années de fréquentation de tous les répondants.

6. L'eau de mer pénètre dans ce polder par une écluse.

\section{RÉSUMÉS}

En Europe occidentale, les premières «dépoldérisations » remontent aux années 1980. Leurs impacts physiques restent insuffisamment étudiés, mais c'est encore plus vrai des réactions sociales qu'engendre ce retour de la mer. Nous avons ainsi étudié la perception de la dépoldérisation dans le bassin d'Arcachon, où une brèche, qui s'est accidentellement formée dans la digue frontale du domaine de Graveyron, pourrait être maintenue par le Conservatoire du Littoral. L'hostilité au maintien de cette brèche est forte parmi la population locale et les promeneurs interrogés dans le site. Cela s'expliquerait par les très faibles connaissances environnementales du public sur le milieu des marais et les impacts des poldérisations ou des dépoldérisations. On constate par ailleurs que les personnes les plus hostiles au projet sont celles qui sont les plus âgées et qui résident à proximité du site ou le fréquentent depuis longtemps. 
Maintenir cette brèche et permettre l'extension d'un pré salé ne serait éventuellement accepté qu'à la condition d'améliorer l'information du public et de mieux aménager ce site protégé deux souhaits clairement formulés par les 200 personnes interrogées lors d'une enquête d'opinion.

In western Europe, de-polderisation policy was launched in the 1980's. While physical studies on de-polderisation should be continued, it is equally important to examine the social context favouring or not the return of the sea. Therefore, through an opinion survey carried out amongst 200 persons, we analysed the public perception of a de-polderisation scheme in the Arcachon Basin at the french Atlantic coast. It consists in an accidental breach, which occured in the sea dike of the polder of Graveyron and should be maintained in the future by the Conservatoire du Littoral (a public organism dealing with the french coasts' conservation). However, the survey revealed that the inhabitants and the walkers frequenting the polder show a strong opposition to this de-polderisation. This "negative " reaction could be linked to a lack of knowledge on the marsh environment and on the environmental impacts of reclamation or de-polderisation. Moreover, a profile of those opposed shows that they are relatively old and frequent the Graveyron-polder for a long time or live near it. Thus, maintaining this breach and the expanding saltmarsh formed behind the dike would only be accepted if people could be better informed and this protected area better equiped - as whished by the respondents.

\section{INDEX}

Mots-clés : dépoldérisation, polder, enquête d'opinion, marais Index géographique : France, Arcachon (bassin d'), Graveyron (domaine de)

Keywords : reclaimed land, de-polderisation, swamp, opinion survey

\section{AUTEURS}

\section{CLAIRE MARCADET}

Université Paul-Valéry Motpellier 3, Département de Biologie-Ecologie-Environnement, Route de Mende, 34199 Montpellier cedex 5, France, clmarcad@libertysurf.fr

\section{LYDIE GOELDNER-GIANELLA}

UMR 8586 CNRS PRODIG, Université Paris 1, Institut de Géographie, 191 rue St-Jacques, 75005

Paris cedex, France,

Lydie.Goeldner-Gianella@univ-paris1.fr 